\title{
Insulin resistance in people living with HIV is associated with exposure to thymidine analogues and/or didanosine and prior immunodeficiency
}

\section{Susanne Dam Nielsen ( $\nabla$ sdn@dadlnet.dk )}

Department of Infectious Diseases, Rigshospitalet, Copenhagen University Hospital - Rigshospitalet, Copenhagen, Denmark

\section{Julie Høgh}

Department of Infectious Diseases, Rigshospitalet, Copenhagen University Hospital - Rigshospitalet, Copenhagen, Denmark

\section{Malene Hove-Skovsgaard}

Department of Infectious Diseases, Rigshospitalet, Copenhagen University Hospital - Rigshospitalet, Copenhagen, Denmark

\section{Marco Gelpi}

Department of Infectious Diseases, Rigshospitalet, Copenhagen University Hospital - Rigshospitalet, Copenhagen, Denmark

\section{Anne Marie Reimer Jensen}

Department of Infectious Diseases, Rigshospitalet, Copenhagen University Hospital - Rigshospitalet, Copenhagen, Denmark

Jan Gerstoft

Department of Infectious Diseases, Rigshospitalet, Copenhagen University Hospital - Rigshospitalet, Copenhagen, Denmark

\section{Thomas Benfield}

Department of Infectious Diseases, Copenhagen University Hospital - Amager and Hvidovre, Hvidovre, Denmark

\section{Heidi Storgaard}

Steno Diabetes Center

\section{Research Article}

Keywords: HIV-infection, diabetes, insulin resistance, comorbidity, antiretroviral therapy

Posted Date: February 10th, 2022

DOI: https://doi.org/10.21203/rs.3.rs-1296618/v1 
License: (c) (i) This work is licensed under a Creative Commons Attribution 4.0 International License. Read Full License 


\section{Abstract}

Background: As people living with HIV (PLWH) are growing older, there is increased incidence of metabolic diseases, including diabetes, for which insulin resistance (IR) is a key determinant. In this study, we aimed to investigate risk factors associated with insulin resistance in PLWH.

Methods: We included well-regulated PLWH without hepatitis co-infection, and with available fasting serum insulin and plasma glucose $(n=643)$ from the Copenhagen Comorbidity in HIV Infection Study. Insulin resistance was calculated using the homeostasis model assessment of insulin resistance (HOMAIR). We investigated the association between risk factors and high HOMA-IR in a logistic regression model adjusted for age, sex, abdominal obesity, smoking status, and origin. When including use of thymidine analogues and/or didanosine in the model, we also adjusted for time with HIV.

Results: Median [IQR] age of PLWH was (IQR) age was 52 years (46-61), 87\% ( $n=557)$ were male. Median [IQR] insulin resistance was 1.86 [1.23-3.14] mmol/L x mU/L. Risk factors significantly associated with insulin resistance included age, $\mathrm{BMI} \geq 25$, abdominal obesity, use of thymidine analogues and/or didanosine, duration of treatment with antiretroviral therapy, and $C D 4^{+}$nadir $<200$.

Conclusions: Insulin resistance in PLWH is associated with both use of thymidine analogues and/or didanosine and prior immunodeficiency suggesting that increased attention on blood glucose in these patients could be beneficial.

\section{Background}

The population of people living with HIV (PLWH) is growing older(1), and, accordingly, there is an increasing incidence of age-related non-AIDS comorbidities including diabetes(2-5). Insulin resistance is a key determinant of diabetes and has previously been associated with age, overweight, and obesity (6). Especially, abdominal obesity is thought to be associated with a high risk of developing insulin resistance $(7,8)$. In PLWH, exposure to old generation antiretroviral therapy (ART) has been associated with both the development of diabetes and long-lasting alterations of body fat from subcutaneous adipose tissue to visceral adipose tissue (9-12).

In this study, we determined insulin resistance in PLWH and aimed to investigate traditional and HIVspecific risk factors associated with insulin resistance. Additionally, we investigated the potential effect modification of prior exposure to old generation ART.

\section{Methods}

\section{Study population and demographics:}

The Copenhagen Comorbidity in HIV Infection (COCOMO) Study is a non-interventional cohort studythat included PLWH from the greater Copenhagen area. Inclusion criteria were a positive HIV test and age $>18$ 
years. The procedures for recruitment and data collection have been described in detail elsewhere(13). Inclusion and baseline examinations took place from March 2015 to December 2016 where 1099 participants were included. Of these, 949 participants participated in the 2-year-follow-up examination which took place from April 2017 to April 2019. In the present study, we included well-treated participants at the 2-year-follow-up examinations with available fasting serum insulin and plasma glucose, current treatment with ART and HIV RNA < 50 copies $/ \mathrm{mL}$, and absence of co-infection with hepatitis B and C. In total 643 participants were included in the present study.

Hepatitis B virus co-infection was defined as positive hepatitis B virus surface antigen. Hepatitis C virus co-infection was defined as positive hepatitis $\mathrm{C}$ virus RNA.

Ethical approval was obtained by the Regional Ethics Committee of Copenhagen (H-8-2014-004). Written informed consent was obtained from all participants.

At the 2-year-follow-up, a physical exam including anthropometrics and blood pressure was performed by trained clinical staff and questionnaires were used to collect information regarding smoking, medical history, and medication. Fasting blood samples were collected and serum insulin, plasma glucose, lowdensity lipoprotein cholesterol (LDL-C), high-density lipoprotein cholesterol (HDL-C), and total cholesterol were measured. Fasting blood samples were collected after $\geq 8$ hours of fasting. Data regarding HIV infection were obtained from a review of medical charts. Exposure to old generation ART was defined as ever use of thymidine analogues (zidovudine and stavudine) and/or didanosine.

\section{Definition of clinical outcomes:}

In accordance with the original homeostasis model assessment (HOMA), insulin resistance was calculated using the equation: fasting plasma glucose (mmol/L) $x$ fasting serum insulin $(\mathrm{mU} / \mathrm{L}) /$ 22.5(14). Due to the lack of standardized cut-off value for insulin resistance(15), we defined high insulin resistance (high HOMA-IR) as the upper quartile of the HOMA insulin resistance index.

According to WHO guidelines, abdominal obesity was defined as waist-to-hip ratio (WHR) $\geq 0.9$ for men and $\geq 0.85$ for women and BMI was classified as $<18.5$ underweight, 18.5-24.99 normal weight, 2529.99 overweight, and $\geq 30 \mathrm{~kg} / \mathrm{m}^{2}$ obese(16).

\section{Statistics:}

We reported frequency counts and percentages for categorical data and continuous data with means and standard deviations for normal deviates and medians with interquartile ranges (IQR) for variables not normally distributed. Furthermore, we used $\chi 2$ test or Fisher's test was used to compare categorical data between groups, and T-test or Mann-Whitney $\mathrm{U}$ test to compare continuous data between groups. 
We investigated the association between high HOMA-IR and traditional and HIV-specific risk factors using a logistic regression model adjusted for age, sex, BMI category, smoking status (previous/current/never smoker), and origin. Traditional risk factors were included in the adjusted model. Additionally, we tested the association between abdominal obesity and insulin resistance by adding abdominal obesity to the predefined model. Furthermore, HIV-specific risk factors (CD $4^{+}, \mathrm{CD} 4^{+} / \mathrm{CD} 8^{+}$-ratio, $\mathrm{CD} 4^{+}$nadir $<200$, prior exposure to old generation ART and previous AIDS-defining conditions) were investigated by adding them to the model one at a time. When including exposure to old generation ART in the model, we also adjusted for time with HIV.

A possible effect modification by prior exposure to old generation ART on the association between significant risk factors and high HOMA-IR was explored by adding an interaction term to the model.

\section{Results}

Among the included participants, the median (IQR) age was 52 years (46-61), 87\% ( $n=557)$ were male, and $52 \%(n=337)$ had prior exposure to old generation ART, Table 1. Median [IQR] index of IR was 1.86 [1.23-3.14] $\mathrm{mmol} / \mathrm{L} \times \mathrm{mU} / \mathrm{L}$.

\section{Traditional risk factors associated with high HOMA-IR:}

Traditional risk factors significantly associated with high HOMA-IR included age (adjusted OR [aOR] =1.57, per decade older, [95\% Cl 1.29-1.90], $P<0.001)$, BMI 25-29.9 (aOR=2.69 [1.73-4.18], compared to BMI 20-24.9, $P<0.001$ ), $\mathrm{BMI} \geq 30$ (aOR=12.43 [6.81-22.67], compared to $\mathrm{BMI} 20-24.9, P<0.001$ ) and abdominal obesity (aOR=4.93 [2.80-8.68], $P<0.001)$, Figure 1. Other traditional risk factors such as smoking status, origin, and sex were not associated with high HOMA-IR, Table 2.

\section{HIV-specific risk factors associated with high HOMA-IR:}

Exposure to old generation ART (aOR=1.87 [1.06-3.30], $P=0.031)$, nadir $\mathrm{CD} 4^{+}<200$ cells $/ \mu \mathrm{L}(\mathrm{aOR}=1.56$ [1.02-2.38], $P=0.038$ ), and duration of treatment with ART (aOR=1.05 per year [1.02-1.09], $P=0.004$ ) were all significantly associated with high HOMA-IR, Figure 1. Current CD4+ count, CD4+/CD8+-ratio, and previous AIDS defining condition were not associated with high HOMA-IR, Table 2.

\section{Effect modification by prior exposure to old generation ART:}

When investigating effect modification by prior exposure to old generation ART, we found the association between abdominal obesity and high HOMA-IR to be stronger in PLWH with prior exposure to old generation ART (aOR=8.08 [3.25-20.12] vs aOR = 2.68 [1.20-5.97], P-interaction=0.042), Figure 1. We found no effect modification between old generation ART and other risk factors. 


\section{Discussion}

In this study of well-treated PLWH, abdominal obesity, BMI $\geq 25$, exposure to old generation ART, and CD4 ${ }^{+}$ nadir $<200$ cells $/ \mu \mathrm{L}$ was associated with insulin resistance. Furthermore, the association between abdominal obesity and insulin resistance was stronger in PLWH with previous use of old generation ART. This suggests that insulin resistance in PLWH is related to both exposure to old generation ART and prior immunodeficiency.

In the COCOMO study, we have previously reported an 1.7 increased odds of diabetes in PLWH compared to uninfected individuals(4). Insulin resistance is a key determinant in the development of diabetes(17). Among the traditional risk factors, age, $\mathrm{BMI} \geq 25$ and abdominal obesity was associated with insulin resistance in PLWH. These are all well-described risk factors for both insulin resistance and diabetes(6). Especially, abdominal obesity, including increased amounts of visceral fat, is thought to be associated with a high risk of developing insulin resistance $(7,8)$.

Among HIV-specific risk factors, exposure to old generation ART was associated with insulin resistance, even when adjusting for time with HIV. In muscle and adipose tissue old generation ART has been shown to inhibit glucose transporter 4 (GLUT4)(18) that contributes to glucose homeostasis. Accordingly, inhibition of GLUT4 may lead to insulin resistance which may explain the association between old generation ART and insulin resistance. Furthermore, old generation ART has been associated with changes in adipose tissue including lipodystrophy $(11,12)$. Interestingly, the association between abdominal obesity and insulin resistance was stronger in PLWH with prior exposure to old generation ART. This may suggest that the alterations of adipose tissue in PLWH exposed to old generation ART increase the effect of abdominal obesity on insulin resistance. Additionally, the duration of treatment with ART was associated with insulin resistance. This is probably explained by the duration of ART being closely related to exposure to old generation ART.

In addition to old generation ART and duration of ART, CD $4^{+}$nadir $<200$ cells $/ \mu \mathrm{L}$ was associated with insulin resistance. This finding is consistent with other studies reporting lower CD $4^{+}$nadir to be associated with an increased risk of diabetes $(2,19)$. Furthermore, both insulin resistance and prior immunodeficiency have been associated with cardiovascular comorbidity in $\mathrm{PLWH}(20)$.

There were limitations to this study. First, the study is cross-sectional which prevents us from drawing any conclusions regarding causality. Second, since there is no standardized cut-off value for insulin resistance the clinically significant insulin resistance is unknown. Third, we could not compare insulin resistance in PLWH to insulin resistance in uninfected controls because we did not have access to a control population with fasting blood samples. The strengths of the study include the large cohort of welltreated PLWH and the use of fasting blood samples to define insulin resistance.

\section{Conclusion}


In conclusion, in well-treated PLWH with absence of chronic hepatitis B or C infection, insulin resistance was associated with abdominal obesity, exposure to old generation ART, and CD $4^{+}$nadir $<200 \mathrm{cells} / \mu \mathrm{L}$. This may suggest that insulin resistance in PLWH is associated with both prior immunodeficiency and metabolic changes due to exposure to old generation ART. Furthermore, the association between abdominal obesity and insulin resistance was stronger in PLWH with exposure to old generation ART than in PLWH without exposure to these drugs, suggesting old generation ART to have long-lasting effects on abdominal adipose tissue related to insulin resistance. This suggests that special attention on blood glucose in these patients could be beneficial.

\section{Abbreviations}

aOR: Adjusted OR

ART: Antiretroviral therapy

COCOMO Study: Copenhagen Comorbidity in HIV Infection Study

GLUT4: Glucose transporter 4

HDL-C: High-density lipoprotein cholesterol

HOMA: Homeostasis model assessment

IQR: Interquartile range

LDL-C: Low-density lipoprotein cholesterol

PLWH: People living with HIV

\section{Declarations}

\section{Ethics approval and consent to participate:}

Ethical approval was obtained by the Regional Ethics Committee of Copenhagen (H-8-2014-004). The study was performed in accordance with the principles of the Declaration of Helsinki. Written informed consent was obtained from all participants.

\section{Consent for publication:}

Not applicable.

\section{Availability of data and materials:}


The datasets generated during and/or analysed during the current study are available from the corresponding author on reasonable request. Danish legislation does not allow the dataset to be freely available.

\section{Competing interests:}

J.H reports grant from Novo Nordisk Foundation and Righospitalet Research Council. M.H.S, M.G, A.M.R.J and J.G reports no conflicts of interest. T.B reports grants from Novo Nordisk Foundation, grants from Simonsen Foundation, grants and personal fees from GSK, grants and personal fees from Pfizer, personal fees from Boehringer Ingelheim, grants and personal fees from Gilead, personal fees from MSD, personal fees from Astra Zeneca, personal fees from Janssen, grants from Lundbeck Foundation, grants from Kai Hansen Foundation, personal fees from Pentabase ApS, grants from Erik and Susanna Olesen's Charitable Fund, outside the submitted work. H.S reports Steno Collaborative Grant, Novo Nordisk Foundation and advisory board activity for Novo Nordisk. S.D.N. has received unrestricted research grants from Novo Nordisk Foundation, Lundbeck Foundation, Rigshospitalet Research Council. Travelling grants from Gilead. Advisory board activity for Gilead and MSD, all unrelated to this manuscript. All other authors report no conflicts of interest.

\section{Funding:}

The COCOMO two-year follow-up was supported by an unrestricted research grant from Gilead Sciences, Inc, Novo Nordic Foundation and Rigshospitalet Research Council.

\section{Authors' contributions:}

J.H. was responsible for concept, statistical analyses, and drafted the manuscript. M.H.S and M.G. were responsible for concept, statistical analysis and edited the manuscript. A.M.R.J, and H.S were responsible for concept and edited the manuscript. J.G, T.B and S.D.N. were responsible for concept, data collection and edited the manuscript.

\section{Acknowledgements:}

We thank all the study subjects for their participation. We thank the staff at the Departments of Infectious Diseases at Rigshospitalet and Amager and Hvidovre Hospital for their dedicated participation.

\section{References}

1. Smit M, Brinkman K, Geerlings S, Smit C, Thyagarajan K, van Sighem A V., et al. Future challenges for clinical care of an ageing population infected with HIV: A modelling study. Lancet Infect Dis. 2015 
Jul 1;15(7):810-8.

2. Brown TT, Cole SR, Li ; Xiuhong, Kingsley LA, Palella FJ, Riddler SA, et al. Antiretroviral Therapy and the Prevalence and Incidence of Diabetes Mellitus in the Multicenter AIDS Cohort Study [published correction appears in Arch Intern Med. 2005 Nov 28;165(21):2541]. Arch Intern Med. 2005;165(10):1179-84.

3. Hernandez-Romieu AC, Garg S, Rosenberg ES, Thompson-Paul AM, Skarbinski J. Is diabetes prevalence higher among HIV-infected individuals compared with the general population? Evidence from MMP and NHANES 2009-2010. BMJ Open Diabetes Res Care. 2017 Jan 1;5(1):e000304.

4. Høgh J, Gelpi M, Skovsgaard MH, Afzal S, Nordestgaard BG, Gerstoft J, et al.HIV infection is associated with type 2 diabetes mellitus. J Acquir Immune Defic Syndr. 2021;88(4):e32-5.

5. Pedro MN, Rocha GZ, Guadagnini D, Santos A, Magro DO, Assalin HB, et al. Insulin Resistance in HIVPatients: Causes and Consequences. Front Endocrinol. 2018;9:514.

6. Fletcher B, Gulanick M, Lamendola C. Risk factors for type 2 diabetes mellitus. J Cardiovasc Nurs. 2002;16(2):17-23.

7. Westphal SA. Obesity, Abdominal Obesity, and Insulin Resistance. Clin Cornerstone. 2008;9(1):2331.

8. Rönnemaa T, Koskenvuo M, Marniemi J, Koivunen T, Sajantila A, Rissanen A, et al. Glucose Metabolism in Identical Twins Discordant for Obesity. The Critical Role of Visceral Fat 1. J Clin Endocrinol Metab. 1997 Feb 1;82(2):383-7.

9. De Wit S, Sabin CA, Weber R, Worm SW, Reiss P, Cazanave C, et al. Incidence and risk factors for newonset diabetes in HIV-infected patients. Diabetes Care. 2008 Jun;31(6):1224-9.

10. Rasmussen LD, Mathiesen ER, Kronborg G, Pedersen C, Gerstoft J, Obel N. Risk of Diabetes Mellitus in Persons with and without HIV: A Danish Nationwide Population-Based Cohort Study. PLoS One. 2012 Sep 12;7(9):e44575.

11. Gelpi M, Afzal S, Fuchs A, Lundgren J, Knudsen AD, Drivsholm N, et al. Prior exposure to thymidine analogs and didanosine is associated with long-lasting alterations in adipose tissue distribution and cardiovascular risk factors. AIDS. 2019 Mar 15;33(4):675-83.

12. Gelpi $M$, Knudsen AD, Larsen KB, Mocroft $A$, Lebech AM, Lindegaard $B$, et al. Long-lasting alterations in adipose tissue density and adiponectin production in people living with HIV after thymidine analogues exposure. BMC Infect Dis. 2019 Aug 9;19(1):708.

13. Ronit A, Haissman J, Kirkegaard-Klitbo DM, Kristensen TS, Lebech AM, Benfield T, et al. Copenhagen comorbidity in HIV infection (COCOMO) study: a study protocol for a longitudinal, non-interventional assessment of non-AIDS comorbidity in HIV infection in Denmark. BMC Infect dis. $2016 \mathrm{Nov}$ 26;16(1):713.

14. Matthews DR, Hosker JP, Rudenski AS, Naylor BA, Treacher DF, Turner RC. Homeostasis model assessment: insulin resistance and $\beta$-cell function from fasting plasma glucose and insulin concentrations in man. Diabetologia. $1985 \mathrm{Jul} ; 28(7): 412-9$. 
15. Muniyappa R, Lee S, Chen H, Quon MJ. Current approaches for assessing insulin sensitivity and resistance in vivo: Advantages, limitations, and appropriate usage. Am J Physiol - Endocrinol Metab. 2008 Jan;294(1):e15-26.

16. Gelpi M, Afzal S, Lundgren J, Ronit A, Roen A, Mocroft A, et al. Higher risk of abdominal obesity, elevated low-density lipoprotein cholesterol, and hypertriglyceridemia, but not of hypertension, in people living with human immunodeficiency virus (HIV): Results from the copenhagen comorbidity in HIV infection study. Clin Infect Dis. 2018;67(4):579-86.

17. Wu WC, Wei JN, Chen SC, Fan KC, Lin CH, Yang CY, et al. Progression of insulin resistance: A link between risk factors and the incidence of diabetes. Diabetes Res Clin Pract. 2020 Mar 1;161:108050.

18. Murata H, Hruz PW, Mueckler M. Indinavir inhibits the glucose transporter isoform Glut4 at physiologic concentrations. AIDS. 2002 Apr 12;16(6):859-63.

19. Spagnuolo V, Galli L, Poli A, Salpietro S, Gianotti N, Piatti P, et al. Associations of statins and antiretroviral drugs with the onset of type 2 diabetes among HIV-1-infected patients. BMC Infect Dis. 2017 Jan 7;17(1):43.

20. Brener MI, Post WS, Haberlen SA, Zhang L, Palella FJ, Jacobson LP, et al. Comparison of insulin resistance to coronary atherosclerosis in human immunodeficiency virus infected and uninfected men (from the Multicenter AIDS Cohort Study). Am J Cardiol. 2016 Mar 15;117(6):993-1000.

\section{Tables}


Table 1

Variable

Age, years, median (IQR)

Sex (male), $n$ (\%)

Scandinavian or other european origin, $n(\%)$

BMI $\left(\mathrm{kg} / \mathrm{m}^{2}\right)$, median (IQR)
PLWH ( $n=643)$

$52(46,61)$

557 (87)

562 (87)

$25(23,27)$

$$
\begin{aligned}
& \text { Underweight, n (\%) } \\
& \text { Normal weight, n (\%) } \\
& \text { Overweight, n (\%) } \\
& \text { Obese, n (\%) }
\end{aligned}
$$$$
13(2)
$$$$
319(50)
$$$$
234(36)
$$$$
77 \text { (12) }
$$

Abdominal obesity, n (\%)

$372(58)$

Smoking status:

$$
\begin{aligned}
& \text { Current, n (\%) } \\
& \text { Previous, n (\%) } \\
& \text { Never, n (\%) }
\end{aligned}
$$$$
154 \text { (24) }
$$$$
218 \text { (34) }
$$$$
266 \text { (41) }
$$

Hypertension, yes, n (\%)

Transmission mode:

\begin{tabular}{ll}
\hline MSM, n (\%) & $461(72)$ \\
\hline Heterosexual, n (\%) & $140(22)$
\end{tabular}

IDU, $\mathbf{n}(\%)$

$5(0.8)$

Other, $\mathrm{n}(\%)$

$10(1.6)$

Current $\mathrm{CD} 4^{+}$, cells $/ \mu \mathrm{L}$, median (IQR)

$\begin{array}{ll}<200 & 10(1.6) \\ \mathbf{2 0 0 - 3 4 9} & 25(4) \\ \mathbf{3 5 0 - 4 9 9} & 101(16) \\ \geq \mathbf{5 0 0} & 467(73)\end{array}$

CD4 ${ }^{+}$Nadir $<200$, cells $/ \mu \mathrm{L}, \mathrm{n}(\%)$

$\mathrm{CD}^{+} / \mathrm{CD}^{+}$ratio, median (IQR)

$0.9(0.6,1.2)$

Time since HIV diagnosis, years, median (IQR) 
History of AIDS (yes), $\mathrm{n}(\%)$

Time with ART treatment, years, median (IQR)

Exposure to older generation ART, yes, $\mathbf{n}(\%)$

Table 1: Abbreviations: MSM; men who have sex with men, IDU; injecting drug use, ART; combined antiretroviral therapy. Hypertension was defined as systolic blood pressure $\geq 140 \mathrm{mmHg}$ and/or diastolic blood pressure $\geq 90 \mathrm{mmHg}$ and/or antihypertensive treatment.

\section{Table 2}

Risk factors associated with high HOMA-IR 
aOR $[95 \% \mathrm{Cl}]$

$P$-value

Traditional risk factors:

Age, per decade

Male sex

Abdominal obesity

BMI:

Normal weight

Overweight

Obese

Smoking:

Never smoker

Current smoker

Previous smoking

Non-european origin

HIV-specific risk factors

Exposure to old generation ART *

Duration of ART, per year

CD4 ${ }^{+}$count, per 100 cells $/ \mu \mathrm{L}$

$\mathrm{CD}^{+}$nadir $<200$ cells $/ \mu \mathrm{L}$

$\mathrm{CD}^{+} / \mathrm{CD} 8^{+}$-ratio, per 0.1 change in ratio

Previous AIDS defining condition
1.57 [1.29-1.90]

$<0.001$

$1.04[0.57-1.90]$

0.894

$4.93[2.80-8.68]$

$<0.001$

Ref.

$2.69[1.73-4.18]$

$<0.001$

$12.43[6.81-22.67]$

$<0.001$

Table 2: Abbreviations: ART; combined antiretroviral therapy. VAT; visceral adipose tissue. SAT; subcutaneous adipose tissue.

Adjusted OR calculated using logistic regression in a model adjusted for age, sex, BMI, smoking status, and origin.

*Calculated using our adjusted model, further adjusting for time with HIV. 


\section{OR of high levels of insulin resistance}

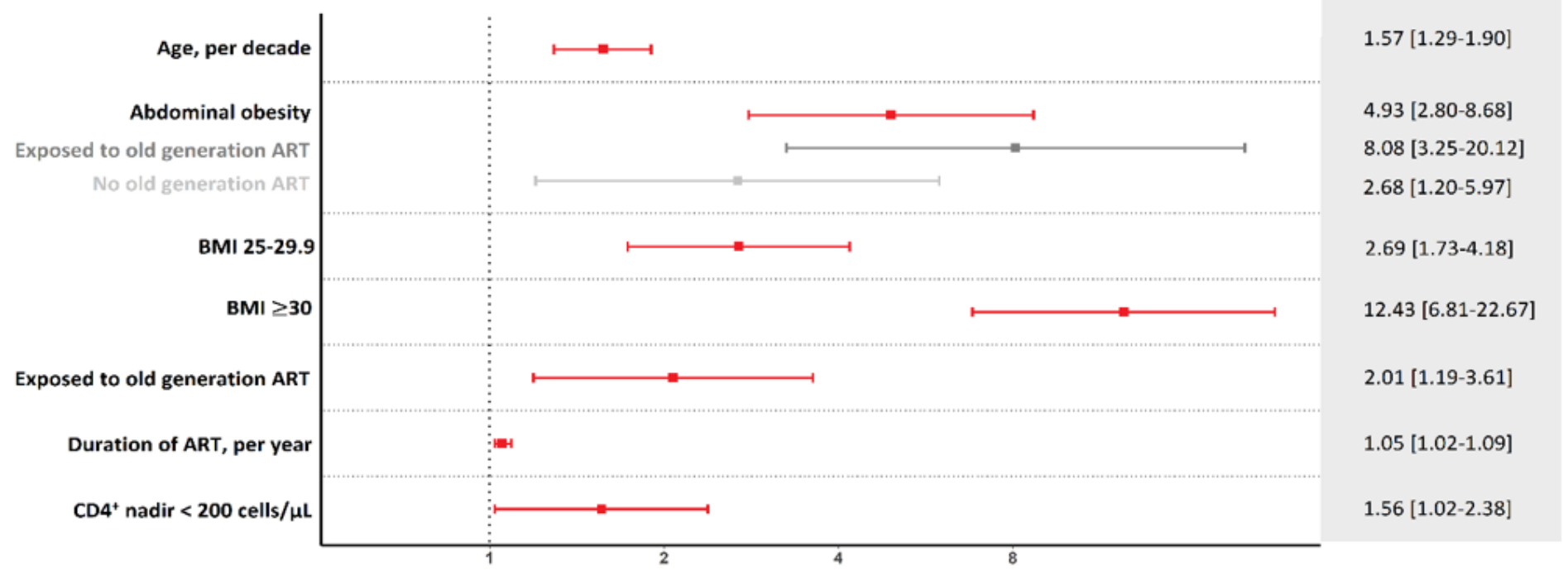

\section{Figure 1}

Adjusted OR calculated using a logistic regression model adjusted for age, sex, BMI, smoking status, and origin. When including exposure to old generation ART in the model we also adjusted for time with HIV. 